\title{
About the Casimir scaling hypothesis
}

\author{
Claude Semay* \\ Groupe de Physique Nucléaire Théorique, Université de Mons-Hainaut, \\ Académie universitaire Wallonie-Bruxelles, Place du Parc 20, B-7000 Mons, Belgium
}

(Dated: November 12, 2018)

\begin{abstract}
A lattice calculation shows that the Casimir scaling hypothesis is well verified in QCD, that is to say that the potential between two opposite color charges in a color singlet is proportional to the value of the quadratic Casimir operator. On the other hand, in a bag model calculation for the same system, a scaling of the string tension with the square root of the quadratic Casimir operator is obtained. It is shown that, within the same formalism but with the assumption that the width of the string is independent of the color charges, the string tension is proportional to value of the quadratic Casimir operator. Some considerations about the color behavior of the total interaction are given.
\end{abstract}

The Casimir scaling hypothesis means that the potential between two opposite colour charges in a colour singlet is proportional to the value of the quadratic Casimir operator. A lattice calculation 1] exclude any violations of this hypothesis that exceed $5 \%$ for charge separations of up to $1 \mathrm{fm}$. Nevertheless, other models do not predict such a colour behaviour. For instance, a scaling of the string tension with the square root of the quadratic Casimir operator is obtained in a bag model calculation 2]. We show here that the Casimir scaling can be obtained if the fundamental assumption in the bag model, the existence of a confining pressure $B$, is replaced by the hypothesis of the existence of a universal string section in the rest frame of the charges.

We use the same formalism as in Ref. 2]. Let us consider two opposite colour charges with zero mass, moving attached by a string, in a colour singlet. The colour electric flux $\vec{E}_{a}$ which leaves a colour charge has the strength

$$
\left|\vec{E}_{a}\right| A=g \lambda_{a}
$$

where $A$ is the cross section of the string, and $\lambda_{a}$ are the colour matrices. If $x$ is the distance from the centre of mass (middle of the string), a point of the string moves with the speed

$$
v=\frac{2}{L} x
$$

where $L$ is the length of the string. The colour charges at the extremities move at the speed of light. The colour magnetic field, which is produced by the rotation of the colour electric field, is given by

$$
\vec{B}_{a}=\vec{v} \times \vec{E}_{a}
$$

at a point of the string which moves with velocity $\vec{v}$. The quadratic Casimir operator $C$ is

$$
C=\frac{1}{4} \sum_{a} \lambda_{a}^{2}
$$

*FNRS Research Associate; E-mail: claude.semay@umh.ac.be
In Ref. 2], the section of the string is determined by the surface equation of the bag containing the coloured particles. This implies that its section $A$ is proportional to $\sqrt{C}$. In this work, we assume that the section of the string is a constant $A_{0}$, independent of $C$, in the rest frame of the string. Consequently, our model is not a bag model, and no confining pressure $B$ is introduced. When the string rotates, the section undergoes a Lorentz contraction

$$
A=A_{0} \sqrt{1-v^{2}}
$$

To calculate the mass $M$ of the colour singlet system, let us first compute the strength fields

$$
\sum_{a} E_{a}^{2}=\frac{4 g^{2} C}{A^{2}} \text { and } \sum_{a} B_{a}^{2}=\frac{4 g^{2} C}{A^{2}} v^{2},
$$

the speed $\vec{v}$ of a point of the string being always perpendicular to $\vec{E}_{a}$. All volume integrals are replaced by

$$
\int d^{3} x \rightarrow 2 \int_{0}^{L / 2} A d x=L \int_{0}^{1} A d v .
$$

The energy of the coloured flux lines is [2]

$$
E_{f}=\frac{1}{2} \int d^{3} x \sum_{a}\left(E_{a}^{2}+B_{a}^{2}\right) .
$$

With the notations defined above, we obtain

$$
E_{f}=2 g^{2} C \frac{L}{A_{0}} \int_{0}^{1} \frac{1+v^{2}}{\sqrt{1-v^{2}}} d v .
$$

The angular momentum of the coloured flux lines is 2

$$
\vec{J}_{f}=\int d^{3} x \sum_{a} \vec{r} \times\left(\vec{E}_{a} \times \vec{B}_{a}\right) .
$$

Thus, we obtain

$$
J_{f}=2 g^{2} C \frac{L^{2}}{A_{0}} \int_{0}^{1} \frac{v^{2}}{\sqrt{1-v^{2}}} d v .
$$


Classically, a massless colour charge does not carry nor energy neither momentum [3]. Consequently, the mass $M$ of the state is equal to $E_{f}$ and the total angular momentum $J$ is equal to $J_{f}$. We then obtain

$$
M^{2}=\frac{9 \pi}{2} \frac{g^{2}}{A_{0}} C J=18 \pi^{2} \frac{\alpha_{S}}{A_{0}} C J
$$

with $\alpha_{S}=g^{2} / 4 \pi$ the strong coupling constant. Let us note that, in Ref. [2], the mass is determined from the condition $\partial M / \partial L=0$. But this implies also that the contributions of the massless colour charges to energy and momentum are vanishing.

We obtain the linear Regge trajectories, but with a slope-that is to say a string tension-proportional to $C$, and not to $\sqrt{C}$. This result has already been obtained in Ref. [4], but with a different technique. With the more phenomenological approach used here, we find that the energy density of the flux tube is given by

$$
\frac{M}{L}=6 \pi^{2} \frac{\alpha_{S} C}{A_{0}},
$$

which is quite different from the result of Ref. [4].

In order to check the relevance of formula (12), let us consider the case of a meson, for which $C=4 / 3$. The relativistic flux tube model [3] predict that

$$
M^{2}=2 \pi a J
$$

where $a$ is the usual string tension. It is then possible to link the section $A_{0}$ of the string to its tension $a$ and the strong coupling constant $\alpha_{S}$

$$
A_{0}=12 \pi \frac{\alpha_{s}}{a}
$$

The radius $R_{0}$ of the string is given by $\sqrt{A_{0} / \pi}$, assuming a cylindrical form for the string. For reasonable values of the QCD parameters, $\alpha_{S} \in[0.1-0.4]$ and $a \in[0.17-$ $0.20] \mathrm{GeV}^{2}$, we find $R_{0}$ in the range $0.5-1.0 \mathrm{fm}$ [4]. A lattice calculation predicts a gaussian string width with a mean radius around $0.35 \mathrm{fm}$ [5]. Given the simplicity of our model, the agreement is quite reasonable.

We can expect that our model is relevant only if $L>$ $2 R_{0}$. This condition is satisfied if

$$
J>8 \pi C \alpha_{s}
$$

Small values for $J$ are acceptable if the product $C \alpha_{S}$ is not too large.

The key ingredient of this work is the assumption that the width of the string is independent of the colour charges. Such a possibility is also studied in recent works 6, 7]. It could be interesting to test this hypothesis with lattice calculations.

Besides the confinement, a one-gluon exchange process exists between the two particles. The colour dependence of this interaction is given by

$$
\frac{1}{4} \sum_{a} \lambda_{a}(1) \lambda_{a}(2)=\frac{1}{2}(0-C-C)=-C .
$$

So we find again a colour scaling given by $C$. A constant potential plays an important role in the hadron spectroscopy. In various approaches [8, 9], this constant is proportional to the string tension. In this case the colour scaling is also given by $C$. Finally, we can expect that the total potential between two opposite colour charges in a colour singlet is proportional to the quadratic Casimir operator, and not to its square root.
[1] G. S. Bali, Phys. Rev. D 62, 114503 (2000) hep-lat/0006022.

[2] K. Johnson and C. B. Thorn, Phys. Rev. D 13, 1934 (1976).

[3] D. LaCourse and M. G. Olsson, Phys. Rev. D 39, 2751 (1989).

[4] T. H. Hansson, Phys. Lett. B 166, 343 (1986).

[5] G. S. Bali, C. Schlichter, and K. Schilling, Phys. Rev. D 51, 5165 (1995) hep-lat/9409005.
[6] B. Lucini and M. Teper, Phys. Rev. D 64, 105019 (2001) hep-lat/0107007.

[7] A. I. Shoshi, F. D. Steffen, H. G. Dosch, and H. J. Pirner, Phys. Rev. D 68, 074004 (2003) hep-ph/0211287.

[8] D. Gromes, Z. Phys. C 11, 147 (1981); erratum 14, 94 (1982).

[9] Yu. A. Simonov, Phys. Lett. B 515, 137 (2001) hep-ph/0105141. 